\title{
Behaviour of two-compartment models
}

\author{
Jianfeng Feng ${ }^{\mathrm{a}, \mathrm{b}, *, 1}$, Guibin $\mathrm{Li}^{\mathrm{b}}$ \\ ${ }^{\mathrm{a}}$ COGS, School of Cognitive and Computing, Sussex University, Brighton, Sussex BN1 9QH, UK \\ ${ }^{\mathrm{b}}$ Computational Neuroscience Laboratory, The Babraham Institute, Cambridge CB2 4AT, UK
}

\begin{abstract}
For two-compartment models: the integrate-and-fire model and the Pinsky-Rinzel model, we show that the smaller the soma is, the faster and the more irregularly the neuron fires. We also find that when the soma is small, two-compartment models can be naturally employed as slope detectors. (C) 2001 Elsevier Science B.V. All rights reserved.

Keywords: Two-compartment integrate-and-fire model; Pinsky-Rinzel model; Coefficient of variation; Slope detector
\end{abstract}

\section{Introduction}

It is well documented in the literature that the geometrical structure of a neuron considerably contributes to its information processing capacity [13]. However, a fully detailed model is usually hard to study theoretically and, most profoundly, the nonhomogeneous distribution of ionic channels along dendritic trees prevents such an investigation. In order to achieve a better understanding of the function of neuronal morphology, we consider two-compartment neuron models which reflect the minimal geometry of a neuron.

In $[16,15]$, the authors have addressed a similar problem using numerical simulations where the model receives constant and deterministic input. Here, we consider the case that neurons receive random, rather than deterministic inputs. The stochastic part of an input signal might play a functional role in processing information, see for example $[10,12]$ and references therein. Moreover, studies in $[16,15]$ are confined to

\footnotetext{
${ }^{1}$ http://www.cogs.susx.ac.uk/users/jianfeng

* Corresponding author. Tel.: + 44-1273-606755; fax: + 44-1273-671320.

E-mail address: jianfeng@cogs.sesxs.ac.uk (J. Feng).
} 
a specific model and numerical simulations, whereas some of our conclusions are obtained for generic two-compartment models using theoretical studies (see our full paper [11] for more details). We find that when the somatic compartment is small, the model tends to burst and the bursting length is totally determined by the activity of dendritic compartment. When the somatic compartment is large, the model can be reduced to a single compartment. Furthermore, we also consider the model with nonconstant, deterministic inputs and find that in this case, the model employs bursting activity to detect the slope of incoming signals.

\section{Models}

Let us assume that a neuron is composed of two compartments: a somatic and dendritic compartments. Suppose that a cell receives EPSPs at $q_{\mathrm{E}}$ excitatory synapses and IPSPs at $q_{\mathrm{I}}$ inhibitory synapses and that $V_{\mathrm{d}}(t)$ is the membrane potential of the dendritic compartment at time $t$. When the somatic membrane potential $V_{\mathrm{s}}(t)$ is between the resting potential $V_{\text {rest }}$ and the threshold $V_{\text {thre }}$ :

$$
\begin{aligned}
& \mathrm{d} V_{\mathrm{s}}(t)=-\frac{1}{\gamma}\left(V_{\mathrm{s}}(t)-V_{\text {rest }}\right) \mathrm{d} t+g_{c} \frac{V_{\mathrm{d}}(t)-V_{\mathrm{s}}(t)}{p} \mathrm{~d} t, \\
& \mathrm{~d} V_{\mathrm{d}}(t)=-\frac{1}{\gamma}\left(V_{\mathrm{d}}(t)-V_{\text {rest }}\right) \mathrm{d} t+g_{c} \frac{V_{\mathrm{s}}(t)-V_{\mathrm{d}}(t)}{1-p} \mathrm{~d} t+\frac{d i_{\text {syn }}(t)}{1-p},
\end{aligned}
$$

where $1 / \gamma$ is the decay rate and $p$ is the ratio between the membrane area of the somatic compartment and the whole cell. $g_{c}>0$ is a constant, and the synaptic input is

$$
i_{\mathrm{syn}}(t)=a \sum_{i=1}^{q_{\mathrm{E}}} \mathrm{d} E_{i}(t)-b \sum_{j=1}^{q_{\mathrm{1}}} \mathrm{d} I_{j}(t)
$$

where $E_{i}(t), I_{i}(t)$ are Poisson processes with rates $\lambda_{\mathrm{E}}$ and $\lambda_{\mathrm{I}}$ respectively and $a, b$ are the magnitudes of each EPSP and IPSP. After $V_{\mathrm{s}}(t)$ crosses $V_{\text {thre }}$ from below, a spike is generated and $V_{s}(t)$ is reset to $V_{\text {rest }}$. This model is called the two-compartment integrate-and-fire (IF) model. The interspike interval of efferent spikes is

$$
T(p)=\inf \left\{t: V_{\mathrm{s}}(t) \geqslant V_{\text {thre }}\right\},
$$

for $1>p>0$. It is well known that Poisson input can be approximated by

$$
I_{\text {syn }}(t)=\mu t+\sigma B_{t},
$$

where $B_{t}$ is the standard Brownian motion, $\mu=a q_{\mathrm{E}} \lambda_{\mathrm{E}}-b q_{\mathrm{I}} \lambda_{\mathrm{I}}$ and $\sigma=\sqrt{a^{2} q_{\mathrm{E}} \lambda_{\mathrm{E}}+b^{2} q_{\mathrm{I}} \lambda_{\mathrm{I}}}$. Thus, Eq. (1) now becomes:

$$
\begin{aligned}
& \mathrm{d} V_{\mathrm{s}}(t)=-\frac{1}{\gamma}\left(V_{\mathrm{s}}(t)-V_{\text {rest }}\right) \mathrm{d} t+g_{c} \frac{V_{\mathrm{d}}(t)-V_{\mathrm{s}}(t)}{p} \mathrm{~d} t, \\
& \mathrm{~d} V_{\mathrm{d}}(t)=-\frac{1}{\gamma}\left(V_{\mathrm{d}}(t)-V_{\text {rest }}\right) \mathrm{d} t+g_{c} \frac{V_{\mathrm{s}}(t)-V_{\mathrm{d}}(t)}{1-p} \mathrm{~d} t+\frac{\mathrm{d} I_{\text {syn }}(t)}{1-p} .
\end{aligned}
$$


In the following, we consider the two-compartment IF model to be the model defined by Eq. (2).

We also consider a simplified, two-compartment biophysical model, proposed by Pinsky and Rinzel [16]. They have demonstrated that the model mimics a full, very detailed Traub model quite well.

The Pinsky-Rinzel model is defined by

$$
\begin{aligned}
C_{m} \mathrm{~d} V_{\mathrm{s}}(t)= & -I_{\text {Leak }}\left(V_{\mathrm{s}}\right) \mathrm{d} t-I_{\mathrm{Na}}\left(V_{\mathrm{s}}, h\right) \mathrm{d} t-I_{K-D R}\left(V_{\mathrm{s}}, n\right) \mathrm{d} t \\
& +g_{c} \frac{V_{\mathrm{d}}(t)-V_{\mathrm{s}}(t)}{p} \mathrm{~d} t, \\
C_{m} \mathrm{~d} V_{\mathrm{d}}(t)= & -I_{\text {Leak }}\left(V_{\mathrm{d}}\right) \mathrm{d} t-I_{C a}\left(V_{\mathrm{d}}, s\right) \mathrm{d} t-I_{K-A H P}\left(V_{\mathrm{d}}, q\right) \mathrm{d} t \\
& -I_{K-C}\left(V_{\mathrm{d}}, C a, c\right) \mathrm{d} t+\frac{\mathrm{d} I_{\text {Syn }}}{1-p}+g_{c} \frac{V_{\mathrm{s}}(t)-V_{\mathrm{d}}(t)}{1-p} \mathrm{~d} t, \\
C a^{\prime}=- & 0.002 I_{C a}-0.0125 C a .
\end{aligned}
$$

In our calculations, all parameters and equations are identical to those used in [16] except for the parameters of the calcium equation which are from [17].

\section{Results}

In this section, for both the IF and the Pinsky-Rinzel model, we are going to show that cells falling in between the two limiting cases (large soma or large dendrite as discussed in [11]) form a continuum with respect to their firing properties (mean and coefficient of variation $(\mathrm{CV})$ ), i.e. for $\mathrm{CV}$ and mean firing time, the gap between $p=0$ and 1 is filled in a monotone manner with respect to $p$.

There have been many research activities devoted to the topic of how a neuron processes information via bursting [14], rather than via individual spikes. In particular, there is both experimental and modelling evidence, that suggests that bursting activity might be employed by neurons as a way of detecting the slope of incoming signals. We show that two-compartment neuron could be as a slope detector.

\subsection{Small $p$ vs. Large $p$}

We simulate the two-compartment IF model and the Pinsky-Rinzel model with the following synaptic parameters: $a=b=0.5 \mathrm{mV}, \lambda_{\mathrm{E}}=100 \mathrm{~Hz}, \lambda_{\mathrm{I}}=0,10, \cdots, 100 \mathrm{~Hz}$, $q_{\mathrm{E}}=q_{\mathrm{I}}=100$. The threshold for detecting a spike for both the soma and dendrite of the Pinsky-Rinzel model is $30 \mathrm{mV}$ and $g_{c}=2.1$. In the two-compartment IF model, we let $V_{\text {rest }}=0, V_{\text {thre }}=20 \mathrm{mV}, \gamma=20.2$ and $g_{c}=4$.

Numerical results for the membrane potentials of the somatic and dentritic compartments of the two-compartment IF model are presented in Fig. 1 for $p=0.05$ with 

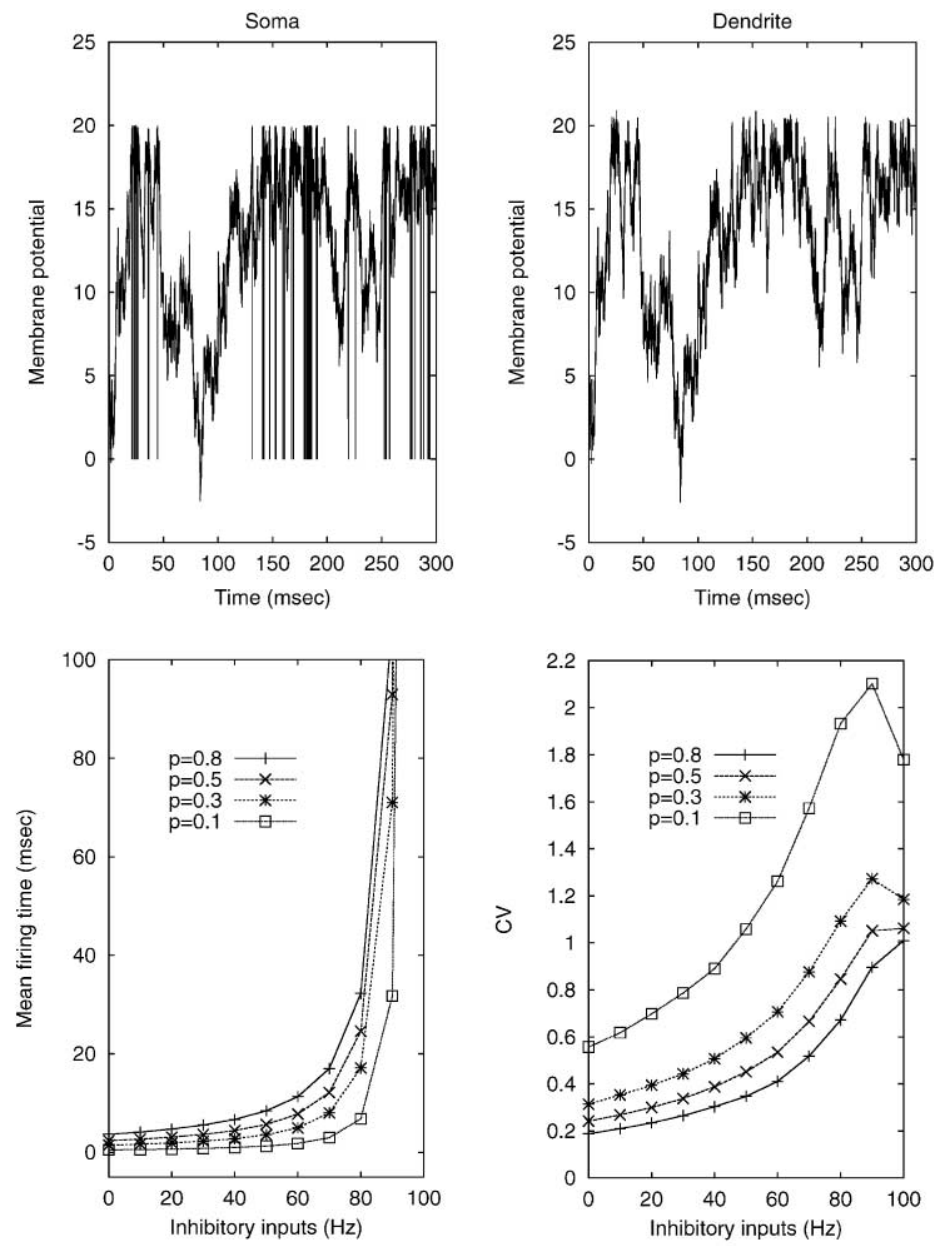

Fig. 1. Upper panel: membrane potential of the two-compartment IF model with $p=0.05$. Note that the membrane potentials of the soma and the dendrite are almost identical, except the reset of the somatic compartment. Bottom panel: Mean firing time and CV vs. $\lambda_{\mathrm{I}}$ for $p=0.1,0.3,0.5,0.8$ for the two-compartment IF model. 10000 spikes are generated for calculating the mean firing time and CV. Parameters are specified in the context.

$\lambda_{\mathrm{I}}=80 \mathrm{~Hz}$. As we have discussed in [11], when $p=0.05$, the model bursts and the bursting behaviour is determined by $V_{\mathrm{d}}$ without coupling. For the two-compartment IF model, as shown in Fig. 1, we see that both the mean firing frequency and the CV are increasing functions of $p$. Therefore the gap between $p=0$ and 1 described in [11] is filled in a monotone manner with respect to $p$. We also note that when $p=0.1$, efferent spike trains are very irregular even when inputs are exclusively excitatory. As we pointed out in Section 2, when $p$ is small, the CV of efferent spike trains is quite high. 
For the Pinsky-Rinzel model, behaviour similar to that of the IF model is observed (see our full paper for more details). The gap between $p=0$ and 1 is filled in a monotone way (see [11]) with respect to $p$. As we adjust the geometrical parameter $p$, the model exhibits a variety of behaviour.

In conclusion, we have obtained a complete picture of the behaviour of these two-compartment models. Both mean firing time and $\mathrm{CV}$ are monotone function of $p$ where $p$ is the ratio of the somatic compartment area with respect to the whole cell.

\subsection{Bursting as a slope detector}

As an application of the results in [11], let us consider the two-compartment IF model with synaptic inputs defined by

$$
\mathrm{d} I_{\text {syn }}(t)=\mu \mathrm{d} t+\cos (\omega t) \mathrm{d} t
$$

where $\omega>0$. For simplicity of notation we assume that $\mu \gamma=V_{\text {thre }}$.

Solving the linear equation

$$
\mathrm{d} V_{\mathrm{d}}=-\frac{V_{\mathrm{d}}}{\gamma} \mathrm{d} t+\mathrm{d} I_{\text {syn }}(t)
$$

with initial condition $V_{\mathrm{d}}(0)=0$ and omitting higher order terms containing $\exp (-t / \gamma)$, we obtain

$$
V_{\mathrm{d}}=V_{\mathrm{thre}}+\gamma \sin (\omega t+\phi),
$$

where $\sin (\phi)=1 /\left(1+\omega^{2} \gamma^{2}\right)$. According to our conclusions in the previous sections, when $p$ is small the neuron will fire with a high frequency (bursting) whenever $\sin (\omega t+\phi)>0$, and will be completely silent if $\sin (\omega t+\phi)<0$. Assume that $\phi$ is small enough so that $\{t: \sin (\omega t+\phi)>0\}$ corresponds to the decreasing part of $\cos (\omega t)$. Then when $p$ is small, the two-compartment IF model is naturally a slope detector, via bursting activities. The presence of $\phi$, a phase lag, in Eq. (5) is a natural constrain for the model behaviour.

When $p$ is large, the model is reduced to the IF model and which has been well studied in the literature [1-12].

In general for the synaptic input:

$$
\mathrm{d} I_{\text {syn }}(t)=s(t) \mathrm{d} t
$$

where $s(t) \in C^{\infty}$, we know that busting could be a detector of the following set:

$$
\left\{t: \gamma s(t)-\gamma^{2} s^{\prime}(t)+\gamma^{3} s^{\prime \prime}(t)-\cdots-V_{\text {thre }} \geqslant 0\right\} .
$$

To further demonstrate our theory above, we simulate the Pinsky-Rinzel model with input (see Fig. 2)

$$
\frac{\mathrm{d} I_{\mathrm{syn}}}{\mathrm{d} t}=\max (\cos (2 \pi t / 200 .)+0.1,0)
$$

Simulation results (not shown) tell us that for parameters $g_{K-D R}=$ 21, $g_{N a}=35, p=0.1$ and 0.9. All other parameters are the same as in Section 2. 


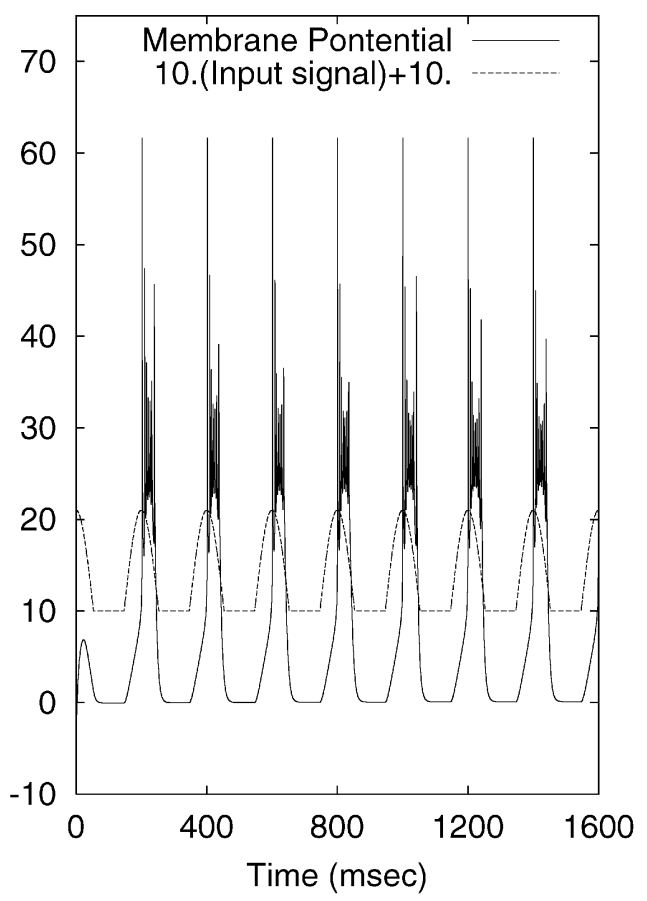

Fig. 2. Membrane potentials of the somatic compartment of the Pinsky-Rinzel model with input signal $\mathrm{d} I_{\text {syn }} / \mathrm{d} t=\max (\cos (2 \pi t / 200)+0.1,0$.$) . Note that the model bursts when the input signal decreases in the$ case where $p=0.1$, and therefore it is a slope detector. When $p$ is large, the neuron fires when it receives a strong signal (not shown).

Then $p=0.1$, the model responds to input signals by emitting bursts. Note that the model bursts only when the input signal decreases, i.e. its slope is negative. When $p=0.9$ the phenomena are not observable at all. It is interesting to note that although the theoretical results above are obtained for a linear model (before resetting) we could generalize it to nonlinear, biophysical models such as the Pinsky-Rinzel model. It seems that the linear theory is widely 'used' by neurons. Furthermore, we point out that the fact that the neuron bursts at the decreasing phase of incoming signals is not trivially due to the delay of neuronal response. In fact, Our results indicate that when the incoming signals reach their minima, the membrane potentials of the somatic compartment also attain their global minima with a delay much shorter than the burst length.

\section{Acknowledgements}

The paper was partially supported by BBSRC and an ESEP of the Royal Society. 


\section{References}

[1] D. Brown, J. Feng, Is there a problem matching model and real CV(ISI)?, Neurocomput. 26-27 (1999) 117-122.

[2] D. Brown, J. Feng, S. Feerick, Varibility of firing of Hodgkin-Huxley and FitzHugh-Nagumo neurons with stochastic synaptic input, Phys. Rev. Lett. 82 (1999) 4731-4734.

[3] J. Feng, Behaviours of spike output jitter in the integrate-and-fire model, Phys. Rev. Lett. 79 (1997) 4505-4508.

[4] J. Feng, Origin of firing varibility of the integrate-and-fire model, Neurocomput. 26-27 (1999) 87-91.

[5] J. Feng, D. Brown, Spike output jitter, mean firing time and coefficient of variation, J. Phys. A 31 (1998) 1239-1252.

[6] J. Feng, D. Brown, Impact of temporal variation and the balance between excitation and inhibition on the output of the perfect integrate-and-fire model, Biol. Cybern. 78 (1998) 369-376.

[7] J. Feng, D. Brown, Coefficient of variation greater than 0.5 how and when?, Biol. Cybern. 80 (1999) 291-297.

[8] J. Feng, D. Brown, Impact of correlated inpus on the output of the integrate-and-fire models, Neural Comput. 12 (2000) 711-732.

[9] J. Feng, D. Brown, Integrate-and-fire models with nonlinear leakage, Bull. Math. Bio. 62 (2000) 467-481.

[10] J. Feng, D. Brown, G. Li, Synchronisation due to common pulsed input in Stein's model, Phys. Rev. E 61 (2000) 2987-2995.

[11] J. Feng, G. Li, Impact of Geometrical Structures on the output of Neuronal Models—From Spikes to Bursts, 2001, submitted.

[12] J. Feng, B. Tirozzi, Stochastic resonance tuned by correlations in neuronal models, Phys. Rev. E. 61 (2000) 4207-4211.

[13] C. Koch, Biophysics of Computation, Oxford University Press, Oxford, 1999.

[14] J.E. Lisman, Bursts as a unit of neural information: making unreliable synapses reliable, Trends Neurosci. 20 (1997) 38-43.

[15] Z.F. Mainen, T.J. Sejnowski, Influence of dendritic structure on firing pattern in model neocortical neuron, Nature 382 (1996) 363-366.

[16] P.F. Pinsky, J. Rinzel, Intrinsic and network rhythmogensi in a reduced Traub model for CA3 neurons, J. Comput. Neurosci. 1 (1994) 39-60.

[17] X.J. Wang, Calcium coding and adaptive temporal computation in cortical pyramidal neurons, J. Neurophysiol. 79 (1998) 1549-1566. 